\title{
Observation of the muon inner bremsstrahlung at LEP1
}

\author{
DELPHI Collaboration
}

J. Abdallah ${ }^{26}$, P. Abreu ${ }^{23}$, W. Adam ${ }^{56}$, P. Adzic ${ }^{12}$, T. Albrecht ${ }^{18}$, R. Alemany-Fernandez ${ }^{9}$, T. Allmendinger ${ }^{18}$, P.P. Allport ${ }^{24}$, U. Amaldi ${ }^{30}$, N. Amapane ${ }^{48}$, S. Amato ${ }^{52}$, E. Anashkin ${ }^{37}$, A. Andreazza ${ }^{29}$, S. Andringa ${ }^{23}$, N. Anjos ${ }^{23}$, P. Antilogus ${ }^{26}$, W-D. Apel ${ }^{18}$, Y. Arnoud ${ }^{15}$, S. Ask ${ }^{9}$, B. Asman ${ }^{47}$, J.E. Augustin ${ }^{26}$, A. Augustinus ${ }^{9}$, P. Baillon ${ }^{9}$, A. Ballestrero ${ }^{49}$, P. Bambade ${ }^{21}$, R. Barbier ${ }^{28}$, D. Bardin ${ }^{17}$, G.J. Barker ${ }^{58}$, A. Baroncelli ${ }^{40}$, M. Battaglia ${ }^{9}$, M. Baubillier ${ }^{26}$, K-H. Becks ${ }^{59}$, M. Begalli ${ }^{7}$, A. Behrmann ${ }^{59}$, E. Ben-Haim ${ }^{21}$, N. Benekos ${ }^{33}$, A. Benvenuti ${ }^{5}$, C. Berat ${ }^{15}$, M. Berggren ${ }^{26}$, D. Bertrand ${ }^{2}$, M. Besancon ${ }^{41}$, N. Besson ${ }^{41}$, D. Bloch ${ }^{10}$, M. Blom $^{32}$, M. Bluj $^{57}$, M. Bonesini ${ }^{30}$, M. Boonekamp ${ }^{41}$, P.S.L. Booth ${ }^{24, b}$, G. Borisov ${ }^{22}$, O. Botner $^{53}$, B. Bouquet ${ }^{21}$, T.J.V. Bowcock ${ }^{24}$, I. Boyko $^{17}$, M. Bracko ${ }^{44}$, R. Brenner ${ }^{53}$, E. Brodet ${ }^{36}$, P. Bruckman ${ }^{19}$, J.M. Brunet ${ }^{8}$, B. Buschbeck ${ }^{56}$, P. Buschmann $^{59}$, M. Calvi ${ }^{30}$, T. Camporesi $^{9}$, V. Canale ${ }^{39}$, F. Carena ${ }^{9}$, N. Castro ${ }^{23}$, F. Cavallo ${ }^{5}$, M. Chapkin ${ }^{43}$, Ph. Charpentier $^{9}$, P. Checchia $^{37}$, R. Chierici $^{9}$, P. Chliapnikov ${ }^{43}$, J. Chudoba ${ }^{9}$, S.U. Chung $^{9}$, K. Cieslik $^{19}$, P. Collins ${ }^{9}$, R. Contri $^{14}$, G. Cosme $^{21}$, F. Cossutti ${ }^{50}$, M.J. Costa ${ }^{54}$, D. Crennell ${ }^{38}$, J. Cuevas ${ }^{35}$, J. D'Hondt ${ }^{2}$, T. da Silva ${ }^{52}$, W. Da Silva ${ }^{26}$, G. Della Ricca ${ }^{50}$, A. De Angelis ${ }^{51}$, W. De Boer ${ }^{18}$, C. De Clercq ${ }^{2}$, B. De Lotto ${ }^{51}$, N. De Maria ${ }^{48}$, A. De Min ${ }^{37}$, L. de Paula ${ }^{52}$, L. Di Ciaccio ${ }^{39}$, A. Di Simone ${ }^{40}$, K. Doroba ${ }^{57}$, J. Drees ${ }^{59,9}$, G. Eigen ${ }^{4}$, T. Ekelof ${ }^{53}$, M. Ellert ${ }^{53}$, M. Elsing ${ }^{9}$, M.C. Espirito Santo ${ }^{23}$, G. Fanourakis ${ }^{12}$, D. Fassouliotis ${ }^{12,3}$, M. Feindt ${ }^{18}$, J. Fernandez ${ }^{42}$, A. Ferrer $^{54}$, F. Ferro ${ }^{14}$, U. Flagmeyer ${ }^{59}$, H. Foeth ${ }^{9}$, E. Fokitis ${ }^{33}$, F. Fulda-Quenzer ${ }^{21}$, J. Fuster ${ }^{54}$, M. Gandelman ${ }^{52}$, C. Garcia ${ }^{54}$, Ph. Gavillet ${ }^{9}$, E. Gazis ${ }^{33}$, R. Gokieli ${ }^{9,57}$, B. Golob ${ }^{44,46}$, G. Gomez-Ceballos ${ }^{42}$, P. Goncalves ${ }^{23}$, E. Graziani $^{40}$, G. Grosdidier ${ }^{21}$, K. Grzelak ${ }^{57}$, J. Guy ${ }^{38}$, C. Haag ${ }^{18}$, A. Hallgren ${ }^{53}$, K. Hamacher ${ }^{59}$, K. Hamilton ${ }^{36}$, S. Haug ${ }^{34}$, F. Hauler ${ }^{18}$, V. Hedberg ${ }^{27}$, M. Hennecke ${ }^{18}$, H. Herr ${ }^{9, b}$, J. Hoffman ${ }^{57}$, S-O. Holmgren ${ }^{47}$, P.J. Holt ${ }^{9}$, M.A. Houlden ${ }^{24}$, J.N. Jackson ${ }^{24}$, G. Jarlskog ${ }^{27}$, P. Jarry ${ }^{41}$, D. Jeans ${ }^{36}$, E.K. Johansson ${ }^{47}$, P. Jonsson ${ }^{28}$, C. Joram ${ }^{9}$, L. Jungermann ${ }^{18}$, F. Kapusta ${ }^{26}$, S. Katsanevas $^{28}$, E. Katsoufis ${ }^{33}$, G. Kernel ${ }^{44}$, B.P. Kersevan ${ }^{44,46}$, U. Kerzel ${ }^{18}$, B.T. King ${ }^{24}$, N.J. Kjaer ${ }^{9}$, P. Kluit ${ }^{32}$, P. Kokkinias ${ }^{12}$, C. Kourkoumelis ${ }^{3}$, O. Kouznetsov ${ }^{17}$, Z. Krumstein ${ }^{17}$, M. Kucharczyk ${ }^{19}$, J. Lamsa $^{1}$, G. Leder ${ }^{56}$, F. Ledroit ${ }^{15}$, L. Leinonen ${ }^{47}$, R. Leitner ${ }^{31}$, J. Lemonne ${ }^{2}$, V. Lepeltier ${ }^{21}$, T. Lesiak ${ }^{19}$, W. Liebig ${ }^{59}$, D. Liko ${ }^{56}$, A. Lipniacka ${ }^{47}$, J.H. Lopes ${ }^{52}$, J.M. Lopez ${ }^{35}$, D. Loukas ${ }^{12}$, P. Lutz ${ }^{41}$, L. Lyons ${ }^{36}$, J. MacNaughton ${ }^{56}$,

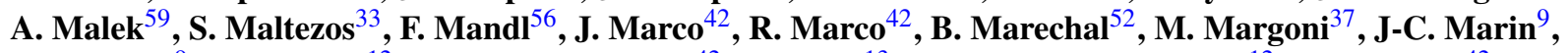
C. Mariotti $^{9}$, A. Markou $^{12}$, C. Martinez-Rivero ${ }^{42}$, J. Masik ${ }^{13}$, N. Mastroyiannopoulos ${ }^{12}$, F. Matorras ${ }^{42}$,

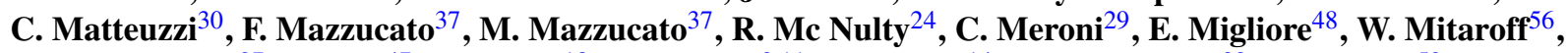
U. Mjoernmark ${ }^{27}$, T. Moa $^{47}$, M. Moch ${ }^{18}$, K. Moenig ${ }^{9,11}$, R. Monge ${ }^{14}$, J. Montenegro ${ }^{32}$, D. Moraes ${ }^{52}$, S. Moreno ${ }^{23}$,

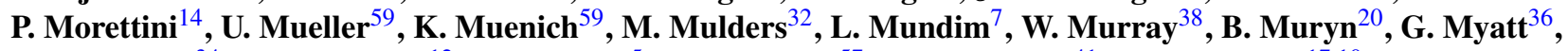
T. Myklebust $^{34}$, M. Nassiakou ${ }^{12}$, F. Navarria ${ }^{5}$, K. Nawrocki ${ }^{57}$, R. Nicolaidou ${ }^{41}$, M. Nikolenko ${ }^{17,10}$, A. Oblakowska-Mucha ${ }^{20}$, V. Obraztsov ${ }^{43}$, A. Olshevski $^{17}$, A. Onofre ${ }^{23}$, R. Orava ${ }^{16}$, K. Osterberg ${ }^{16}$, A. Ouraou $^{41}$, A. Oyanguren ${ }^{54}$, M. Paganoni ${ }^{30}$, S. Paiano ${ }^{5}$, J.P. Palacios ${ }^{24}$, H. Palka ${ }^{19}$, Th.D. Papadopoulou ${ }^{33}$, L. Pape , $^{9}$ C. Parkes $^{25}$, F. Parodi $^{14}$, U. Parzefall ${ }^{9}$, A. Passeri ${ }^{40}$, O. Passon ${ }^{59}$, L. Peralta ${ }^{23}$, V. Perepelitsa ${ }^{54,55}$, A. Perrotta ${ }^{5}$, A. Petrolini ${ }^{14}$, J. Piedra ${ }^{42}$, L. Pieri ${ }^{40}$, F. Pierre ${ }^{41}$, M. Pimenta ${ }^{23}$, E. Piotto ${ }^{9}$, T. Podobnik ${ }^{44,46}$, V. Poireau ${ }^{9}$, M.E. Pol $^{6}$, G. Polok ${ }^{19}$, V. Pozdniakov ${ }^{17}$, N. Pukhaeva ${ }^{17}$, A. Pullia ${ }^{30}$, J. Rames $^{13}$, A. Read $^{34}$, P. Rebecchi ${ }^{9}$, J. Rehn ${ }^{18}$, D. Reid ${ }^{32}$, R. Reinhardt $^{59}$, P. Renton ${ }^{36}$, F. Richard ${ }^{21}$, J. Ridky ${ }^{13}$, M. Rivero ${ }^{42}$, D. Rodriguez ${ }^{42}$, A. Romero $^{48}$, P. Ronchese $^{37}$, P. Roudeau ${ }^{21}$, T. Rovelli ${ }^{5}$, V. Ruhlmann-Kleider ${ }^{41}$, D. Ryabtchikov ${ }^{43}$, A. Sadovsky ${ }^{17}$, L. Salmi ${ }^{16}$, J. Salt ${ }^{54}$, C. Sander $^{18}$, A. Savoy-Navarro ${ }^{26}$, U. Schwickerath ${ }^{9}$, R. Sekulin ${ }^{38}$, M. Siebel ${ }^{59}$, A. Sisakian ${ }^{17}$, G. Smadja $^{28}$, O. Smirnova ${ }^{27}$, A. Sokolov ${ }^{43}$, A. Sopczak ${ }^{22}$, R. Sosnowski ${ }^{57}$, T. Spassov ${ }^{9}$, M. Stanitzki ${ }^{18}$, A. Stocchi ${ }^{21}$, J. Strauss ${ }^{56}$, B. Stugu ${ }^{4}$, M. Szczekowski ${ }^{57}$, M. Szeptycka ${ }^{57}$, T. Szumlak ${ }^{20}$, T. Tabarelli ${ }^{30}$, F. Tegenfeldt ${ }^{53}$, J. Timmermans ${ }^{32, a}$,

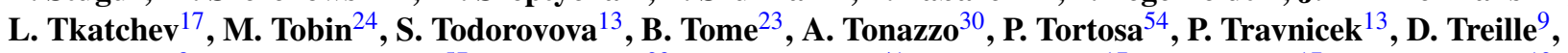
G. Tristram ${ }^{8}$, M. Trochimczuk ${ }^{57}$, C. Troncon ${ }^{29}$, M-L. Turluer ${ }^{41}$, I.A. Tyapkin ${ }^{17}$, P. Tyapkin ${ }^{17}$, S. Tzamarias $^{12}$, V. Uvarov ${ }^{43}$, G. Valenti ${ }^{5}$, P. Van Dam ${ }^{32}$, J. Van Eldik ${ }^{9}$, N. van Remortel ${ }^{16}$, I. Van Vulpen ${ }^{9}$, G. Vegni $^{29}$, F. Veloso $^{23}$, W. Venus ${ }^{38}$, P. Verdier $^{28}$, V. Verzi ${ }^{39}$, D. Vilanova ${ }^{41}$, L. Vitale ${ }^{50}$, V. Vrba ${ }^{13}$, H. Wahlen ${ }^{59}$, A.J. Washbrook ${ }^{24}$, C. Weiser ${ }^{18}$, D. Wicke ${ }^{9}$, J. Wickens ${ }^{2}$, G. Wilkinson ${ }^{36}$, M. Winter ${ }^{10}$, M. Witek ${ }^{19}$, O. Yushchenko ${ }^{43}$, A. Zalewska ${ }^{19}$, P. Zalewski ${ }^{57}$, D. Zavrtanik ${ }^{45}$, V. Zhuravlov ${ }^{17}$, N.I. Zimin ${ }^{17}$, A. Zintchenko ${ }^{17}$, M. Zupan ${ }^{12}$ 
${ }^{1}$ Department of Physics and Astronomy, Iowa State University, Ames, IA 50011-3160, USA

${ }^{2}$ IIHE, ULB-VUB, Pleinlaan 2, 1050 Brussels, Belgium

${ }^{3}$ Physics Laboratory, University of Athens, Solonos Str. 104, 10680 Athens, Greece

${ }^{4}$ Department of Physics, University of Bergen, Allégaten 55, 5007 Bergen, Norway

${ }^{5}$ Dipartimento di Fisica, Università di Bologna and INFN, Via Irnerio 46, 40126 Bologna, Italy

${ }^{6}$ Centro Brasileiro de Pesquisas Físicas, rua Xavier Sigaud 150, 22290 Rio de Janeiro, Brazil

${ }^{7}$ Inst. de Física, Univ. Estadual do Rio de Janeiro, rua São Francisco Xavier 524, Rio de Janeiro, Brazil

${ }^{8}$ Collège de France, Lab. de Physique Corpusculaire, IN2P3-CNRS, 75231 Paris Cedex 05, France

${ }^{9}$ CERN, 1211 Geneva 23, Switzerland

${ }^{10}$ Institut de Recherches Subatomiques, IN2P3-CNRS/ULP-BP20, 67037 Strasbourg Cedex, France

${ }^{11}$ DESY-Zeuthen, Platanenallee 6, 15735 Zeuthen, Germany

${ }^{12}$ Institute of Nuclear Physics, N.C.S.R. Demokritos, P.O. Box 60228, 15310 Athens, Greece

${ }^{13}$ FZU, Inst. of Phys. of the C.A.S. High Energy Physics Division, Na Slovance 2, 182 21, Prague 8, Czech Republic

${ }^{14}$ Dipartimento di Fisica, Università di Genova and INFN, Via Dodecaneso 33, 16146 Genova, Italy

${ }^{15}$ Institut des Sciences Nucléaires, IN2P3-CNRS, Université de Grenoble 1, 38026 Grenoble Cedex, France

${ }^{16}$ Helsinki Institute of Physics and Department of Physical Sciences, P.O. Box 64, 00014 University of Helsinki, Finland

${ }^{17}$ Joint Institute for Nuclear Research, Dubna, Head Post Office, P.O. Box 79, 101000 Moscow, Russian Federation

${ }^{18}$ Institut für Experimentelle Kernphysik, Universität Karlsruhe, Postfach 6980, 76128 Karlsruhe, Germany

${ }^{19}$ Institute of Nuclear Physics PAN, Ul. Radzikowskiego 152, 31142 Krakow, Poland

${ }^{20}$ Faculty of Physics and Nuclear Techniques, University of Mining and Metallurgy, 30055 Krakow, Poland

${ }^{21}$ Université de Paris-Sud, Lab. de l'Accélérateur Linéaire, IN2P3-CNRS, Bât. 200, 91405 Orsay Cedex, France

${ }^{22}$ School of Physics and Chemistry, University of Lancaster, Lancaster LA1 4YB, UK

${ }^{23}$ LIP, IST, FCUL Av. Elias Garcia, 14-1, 1000 Lisboa Codex, Portugal

${ }^{24}$ Department of Physics, University of Liverpool, P.O. Box 147, Liverpool L69 3BX, UK

${ }^{25}$ Department of Physics and Astronomy, Kelvin Building, University of Glasgow, Glasgow G12 8QQ, UK

${ }^{26}$ LPNHE, IN2P3-CNRS, Univ. Paris VI et VII, Tour 33 (RdC), 4 place Jussieu, 75252 Paris Cedex 05, France

${ }^{27}$ Department of Physics, University of Lund, Sölvegatan 14, 22363 Lund, Sweden

${ }^{28}$ Université Claude Bernard de Lyon, IPNL, IN2P3-CNRS, 69622 Villeurbanne Cedex, France

${ }^{29}$ Dipartimento di Fisica, Università di Milano and INFN-MILANO, Via Celoria 16, 20133 Milan, Italy

${ }^{30}$ Dipartimento di Fisica, Università di Milano-Bicocca and INFN-MILANO, Piazza della Scienza 3, 20126 Milan, Italy

${ }^{31}$ IPNP of MFF, Charles University, Areal MFF, V Holesovickach 2, 180 00, Prague 8, Czech Republic

${ }^{32}$ NIKHEF, Postbus 41882, 1009 DB Amsterdam, The Netherlands

${ }^{33}$ Physics Department, National Technical University, Zografou Campus, 15773 Athens, Greece

${ }^{34}$ Physics Department, University of Oslo, Blindern, 0316 Oslo, Norway

${ }^{35}$ Departamento de la Fisica, Universidad Oviedo, Avda. Calvo Sotelo s/n, 33007 Oviedo, Spain

${ }^{36}$ Department of Physics, University of Oxford, Keble Road, Oxford OX1 3RH, UK

${ }^{37}$ Dipartimento di Fisica, Università di Padova and INFN, Via Marzolo 8, 35131 Padua, Italy

${ }^{38}$ Rutherford Appleton Laboratory, Chilton, Didcot OX11 OQX, UK

${ }^{39}$ Dipartimento di Fisica, Università di Roma II and INFN, Tor Vergata, 00173 Rome, Italy

${ }^{40}$ Dipartimento di Fisica, Università di Roma III and INFN, Via della Vasca Navale 84, 00146 Rome, Italy

${ }^{41}$ DAPNIA/Service de Physique des Particules, CEA-Saclay, 91191 Gif-sur-Yvette Cedex, France

${ }^{42}$ Instituto de Fisica de Cantabria (CSIC-UC), Avda. los Castros s/n, 39006 Santander, Spain

${ }^{43}$ Institute for High Energy Physics, Serpukov P.O. Box 35, Protvino, Moscow Region, Russian Federation

${ }^{44}$ J. Stefan Institute, Jamova 39, 1000 Ljubljana, Slovenia

${ }^{45}$ Laboratory for Astroparticle Physics, University of Nova Gorica, Kostanjeviska 16a, 5000 Nova Gorica, Slovenia

${ }^{46}$ Department of Physics, University of Ljubljana, 1000 Ljubljana, Slovenia

${ }^{47}$ Fysikum, Stockholm University, Box 6730, 11385 Stockholm, Sweden

${ }^{48}$ Dipartimento di Fisica Sperimentale, Università di Torino and INFN, Via P. Giuria 1, 10125 Turin, Italy

${ }^{49}$ INFN, Sezione di Torino and Dipartimento di Fisica Teorica, Università di Torino, Via Giuria 1, 10125 Turin, Italy

${ }^{50}$ Dipartimento di Fisica, Università di Trieste and INFN, Via A. Valerio 2, 34127 Trieste, Italy

${ }^{51}$ Istituto di Fisica, Università di Udine and INFN, 33100 Udine, Italy

${ }^{52}$ Univ. Federal do Rio de Janeiro, C.P. 68528 Cidade Univ., Ilha do Fundão 21945-970, Rio de Janeiro, Brazil

${ }^{53}$ Department of Radiation Sciences, University of Uppsala, P.O. Box 535, 75121 Uppsala, Sweden

${ }^{54}$ IFIC, Valencia-CSIC, and D.F.A.M.N., U. de Valencia, Avda. Dr. Moliner 50, 46100 Burjassot (Valencia), Spain

${ }_{55}^{55}$ ITEP, 117259 Moscow, Russian Federation

${ }^{56}$ Institut für Hochenergiephysik, Österr. Akad. d. Wissensch., Nikolsdorfergasse 18, 1050 Vienna, Austria

${ }^{57}$ Institute Nuclear Studies and University of Warsaw, U1. Hoza 69, 00681 Warsaw, Poland

${ }^{58}$ University of Warwick, Coventry CV4 7AL, UK

${ }^{59}$ Fachbereich Physik, University of Wuppertal, Postfach 100 127, 42097 Wuppertal, Germany

Received: 24 June 2007 / Revised: 5 June 2008 / Published online: 11 November 2008

(C) Springer-Verlag / Società Italiana di Fisica 2008 
Abstract Muon bremsstrahlung photons converted in front of the DELPHI main tracker (TPC) in dimuon events at LEP1 were studied in two photon kinematic ranges: $0.2<E_{\gamma} \leq 1 \mathrm{GeV}$ and transverse momentum with respect to the parent muon $p_{T}<40 \mathrm{MeV} / c$, and $1<E_{\gamma} \leq 10 \mathrm{GeV}$ and $p_{T}<80 \mathrm{MeV} / c$. A good agreement of the observed photon rate with predictions from QED for the muon inner bremsstrahlung was found, contrary to the anomalous soft photon excess that has been observed recently in hadronic $Z^{0}$ decays. The obtained ratios of the observed signal to the predicted level of the muon bremsstrahlung are $1.06 \pm$ $0.12 \pm 0.07$ in the photon energy range $0.2<E_{\gamma} \leq 1 \mathrm{GeV}$ and $1.04 \pm 0.09 \pm 0.12$ in the photon energy range $1<E_{\gamma} \leq 10 \mathrm{GeV}$. The bremsstrahlung dead cone is observed for the first time in the direct photon production at LEP.

\section{Introduction}

Recent observation of anomalous soft photon production in hadronic $Z^{0}$ decays collected in the DELPHI experiment at LEP1 [1] has demonstrated the persistence of the soft photon anomaly found earlier in several fixed target experiments with high energy hadronic beams, [2-7]. The photon kinematic range was defined in [1] as follows: $0.2<E_{\gamma} \leq 1 \mathrm{GeV}, p_{T}<80 \mathrm{MeV} / c$, the $p_{T}$ being the photon transverse momentum with respect to the parent jet direction. Though the reaction $e^{+} e^{-} \rightarrow Z^{0} \rightarrow$ hadrons presents a distinct mechanism of hadron production as compared to [2-7], the observed soft photon production characteristics were found in [1] to be very close to those reported in [2-7], both for the measured production rate and for the observed ratio of the rate to the inner hadronic bremsstrahlung. The latter was expected to be the main source of the direct soft photons in kinematic ranges under study (see [8-10]), while the observed signals were found in [1-7] to be several times higher than the bremsstrahlung predictions. No theoretical explanation of this excess is available so far; reviews of the theoretical approaches to the problem can be found in $[11,12]$ (see also the references [13-33] in [1]).

From the experimental analysis, given a similarity of the soft photon production characteristics in both classes of experiments, the conclusion was drawn in [13] that the excess photons are created during the process of hadronization of quarks, i.e. their origin is strongly restricted to reactions of hadron production. If this ansatz is correct, a good agreement should be found between theory and experiment for

\footnotetext{
a e-mail: jan.timmermans@cern.ch
}

${ }^{\mathrm{b}}$ Deceased. the direct soft photon production in reactions of pure electroweak nature. What is the experimental situation in this field?

The electron inner bremsstrahlung in $e^{+} e^{-}$collisions (initial state radiation, ISR) was an important (and rather inconvenient) effect at LEP, with which all the LEP experiments had to contend. No deviation of the ISR characteristics from those expected from theory was observed, either at $Z^{0}$ or at high energy (see for example the DELPHI studies [14-16]). Therefore the situation with the electron inner bremsstrahlung can be considered as showing a nice agreement between theory and experiment.

On the other hand, tests of QED with the muon inner bremsstrahlung which appears as final state radiation (FSR) in $e^{+} e^{-} \rightarrow \mu^{+} \mu^{-}$events were scarce at LEP. There were only two studies of photon production in $Z^{0} \rightarrow \mu^{+} \mu^{-}$ events at LEP1 [17, 18] and a single study of $e^{+} e^{-} \rightarrow$ $\mu^{+} \mu^{-}$events at LEP2 [19]. ${ }^{1}$ All these studies aimed at the separation of rather hard photons, isolated from the neighbouring muon. So, the DELPHI analysis of final state radiation from muons at LEP1 [17] was restricted to the photon kinematic range of $E_{\gamma}>2 \mathrm{GeV}, \theta_{\mu \gamma}>5^{\circ}$, i.e. to the transverse momenta with respect to the muon direction $p_{T}>174 \mathrm{MeV} / c$. In [19] the minimum value of the angle $\theta_{\mu \gamma}$ was increased up to $15^{\circ}$ (keeping the same photon energy threshold), tripling the minimum photon $p_{T}$. The OPAL analysis at LEP1 [18] used photons of $E_{\gamma}>0.9 \mathrm{GeV}$ and $\theta_{\mu \gamma}>200 \mathrm{mrad}$, i.e. the photon transverse momenta with respect to the muon direction were $p_{T}>179 \mathrm{MeV} / c$. Thus, an analysis of the muon inner bremsstrahlung in the soft photon kinematic range close to that analyzed in [1] is completely missing at LEP. This motivated us to study the reaction

$e^{+} e^{-} \rightarrow Z^{0} \rightarrow \mu^{+} \mu^{-} n \gamma, \quad n \geq 1$

at LEP1 in a photon kinematic range similar to the one analyzed in [1] (with the photon transverse momentum being defined now with respect to the parent muon direction). In addition to the low energy (LE) band of $0.2<E_{\gamma} \leq 1 \mathrm{GeV}$ explored in [1], a higher energy (HE) band of $1<E_{\gamma} \leq$ $10 \mathrm{GeV}$ was also used in the analysis, being restricted however to the photons of small transverse momentum with respect to the parent muon direction, $p_{T}<80 \mathrm{MeV} / c$. The $p_{T}$ range of the LE band chosen for the definition of the bremsstrahlung signal was taken narrower in this work as compared to that in [1], namely $p_{T}<40 \mathrm{MeV} / c$. This choice was motivated by the fact that the photon angular variable used in this analysis, the photon polar angle relative to the parent muon direction, can be measured much more

\footnotetext{
${ }^{1}$ Outside the LEP experiments, a few studies of the muon inner bremsstrahlung have been done, see $[20,21]$ and references therein.
} 
accurately as compared to the angular variable used in [1], the photon polar angle relative to the parent jet direction, and this confined most of the LE bremsstrahlung photons down to the mentioned $p_{T}$ range.

The results obtained in this study are presented both uncorrected and corrected for the photon detection efficiency. The presentation of the uncorrected results is motivated by their better statistical accuracies and smaller systematic uncertainties in the absolute photon rates.

\section{Theoretical predictions for the muon inner bremsstrahlung}

In electroweak reactions like (1) the inner bremsstrahlung is a process of direct photon production calculated via purely QED machinery. The production rates for the bremsstrahlung photons from colliding $e^{+} e^{-}$(ISR) and from final $\mu^{+} \mu^{-}$pairs (FSR) in the $p_{T}$ range under study can be calculated at once using a universal formula descending from Low [9] with a modification suggested by Haissinski [22]:

$$
\frac{d N_{\gamma}}{d^{3} \vec{k}}=\frac{\alpha}{(2 \pi)^{2}} \frac{1}{E_{\gamma}} \int d^{3} \vec{p}_{\mu} \sum_{i, j} \eta_{i} \eta_{j} \frac{\left(\vec{p}_{i \perp} \cdot \vec{p}_{j \perp}\right)}{\left(P_{i} K\right)\left(P_{j} K\right)} \frac{d N_{\mu}}{d^{3} \vec{p}_{\mu}}
$$

where $K$ and $\vec{k}$ denote photon four- and three-momenta, $P$ are the 4-momenta of the beam $e^{+}, e^{-}$and the muon involved, and $\vec{p}_{\mu}$ is the 3-momentum of the muon; $\vec{p}_{i \perp}=\vec{p}_{i}-$ $\left(\vec{n} \cdot \vec{p}_{i}\right) \cdot \vec{n}$ and $\vec{n}$ is the photon unit vector, $\vec{n}=\vec{k} / k ; \eta=1$ for the beam electron and for the outgoing $\mu^{+}, \eta=-1$ for the beam positron and for the outgoing $\mu^{-}$, and the sum is extended over both beam particles and the parent muon (formula (2) is presented in the form of the photon production rate per muon); the last factor in the integrand is a differential production rate of the parent muon.

As can be seen, formula (2) is of the lowest (leading) order in $\alpha$. Higher order radiative corrections to it can be evaluated using exponentiated photon spectra in the LE and HE bands. In the accepted regions of low $p_{T}$ the effects of the exponentiation were found to be rather small, as considered in Sect. 6.2.

To a great extent, formula (2) is used in this paper specifically to enable a comparison with the corresponding formula applied for the calculation of the inner hadronic bremsstrahlung in hadronic decays of $Z^{0}$ [1] (cf. the analogous formulae in [2-7]):

$$
\begin{aligned}
\frac{d N_{\gamma}}{d^{3} \vec{k}}= & \frac{\alpha}{(2 \pi)^{2}} \frac{1}{E_{\gamma}} \int d^{3} \vec{p}_{1} \ldots d^{3} \vec{p}_{N} \\
& \times \sum_{i, j} \eta_{i} \eta_{j} \frac{\left(\vec{p}_{i \perp} \cdot \vec{p}_{j \perp}\right)}{\left(P_{i} K\right)\left(P_{j} K\right)} \frac{d N_{h}}{d^{3} \vec{p}_{1} \ldots d^{3} \vec{p}_{N}}
\end{aligned}
$$

where $K$ and $\vec{k}$ denote again photon four- and threemomenta, $P$ are the 4-momenta of the beam $e^{+}, e^{-}$and $N$ charged outgoing hadrons, and $\vec{p}_{1} \ldots \vec{p}_{N}$ are the 3-momenta of the hadrons; $\eta=1$ for the beam electron and for positive outgoing hadrons, $\eta=-1$ for the beam positron and negative outgoing hadrons, and the sum is extended over all the $N+2$ charged particles involved; the last factor in the integrand is a differential hadron production rate (when calculating the photon production rate per jet only hadrons lying in the forward hemisphere of a given jet enter the sum). Calculations performed with formulae (2), (3) show that the inner bremsstrahlung rate from one muon is approximately equal, in the kinematic region under study, to the predicted inner hadronic bremsstrahlung from a whole hadronic jet of a $Z^{0}$ hadronic decay. To a great extent, this is a consequence of the coherence of the photon radiation from the individual radiation sources, the charged hadrons produced in the fragmentation process.

The contribution of the ISR to these rates is small, being below $1 \%$ in the photon kinematic range chosen for the analysis. This smallness is easy to understand: although the ISR from electron/positron beams is much more intense than the ISR from hadron beams in experiments [2-7], where it contributed a significant amount to the detected photon rate, all the extra photons in an experiment with colliding $e^{+} e^{-}$are emitted at very small polar angles with respect to the beam directions, with the angular distribution peaking at $\Theta_{\gamma}=\sqrt{3} / \Gamma$, where $\Gamma$ is a beam Lorentz factor $\left(\Gamma=0.89 \times 10^{5}\right.$ at the $Z^{0}$ peak), thus yielding few photons in the barrel region used in our analysis.

The muon bremsstrahlung radiation (FSR) has the same angular behaviour of the photon production rate versus the photon polar angle relative to the parent muon direction (the photon production angle, $\theta_{\gamma}$ ), with $\Gamma$ being in this case a muon Lorentz factor. For the muons from $Z^{0}$ decays at rest the $\Gamma=4.3 \times 10^{2}$ corresponds to the peak position at $4.0 \mathrm{mrad}$. Note that the position of the peak does not depend on the bremsstrahlung photon energy, since the dependences of the photon production rate on the photon energy and the photon production angle are factorized in formulae (2), (3). The turnover of the muon bremsstrahlung angular distribution at the peak value and its vanishing at $\theta_{\gamma} \rightarrow 0$ is termed the dead cone effect. This behaviour is illustrated by Fig. 1a where the initial part of the production angle distribution for the FSR of the reaction (1) is shown, generated ${ }^{2}$ with formula (2). The observation of the dead cone presents an experimental challenge requiring a highly accurate apparatus; the angular resolution of the opening angle between the measured muon and photon directions which is necessary for the observation of the muon bremsstrahlung dead cone at LEP1, has to be of the order of $1-2 \mathrm{mrad}$.

\footnotetext{
${ }^{2}$ The Monte Carlo data set of dimuon events described below was used as the input of the generation.
} 


\section{DELPHI}
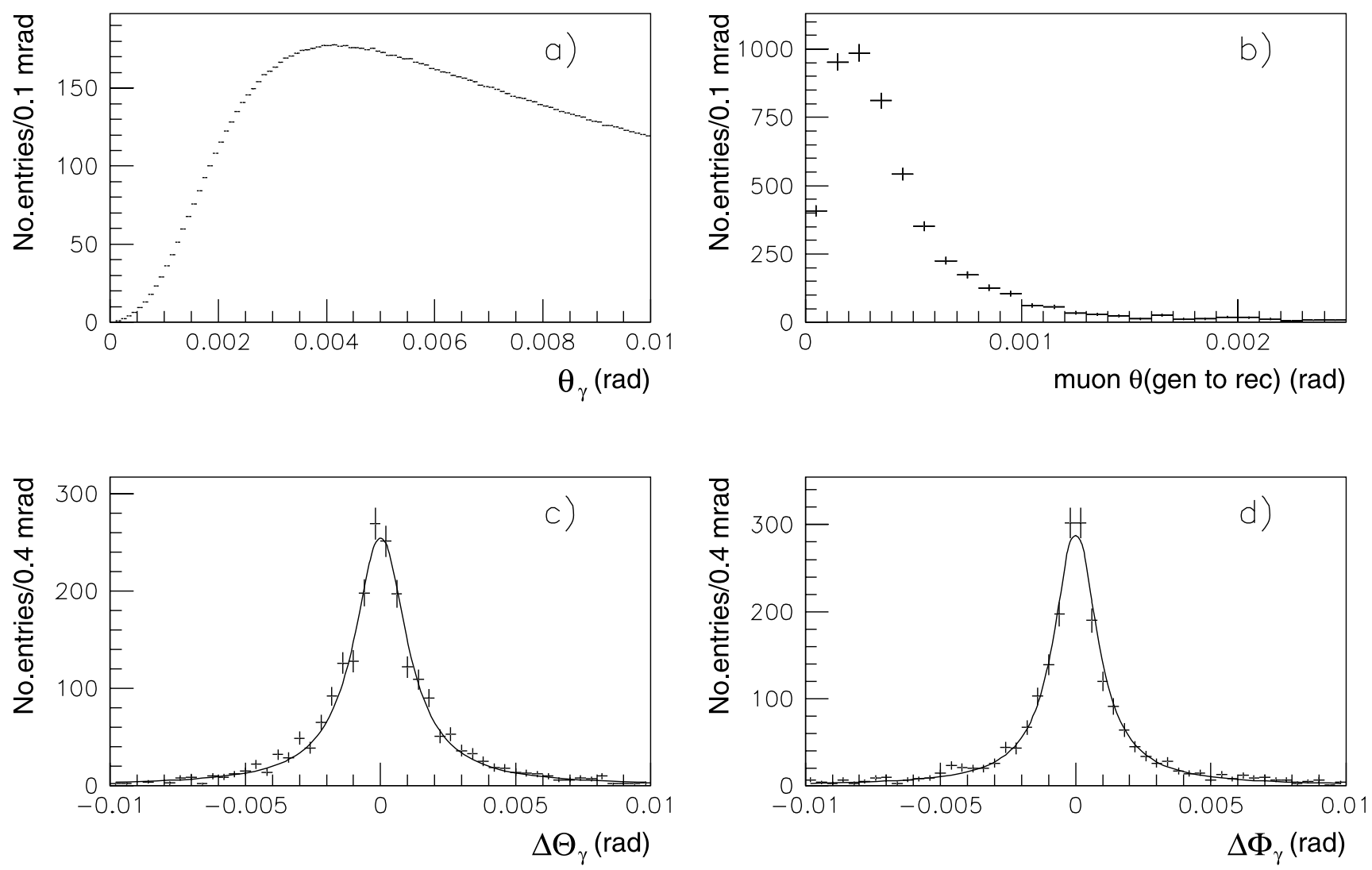

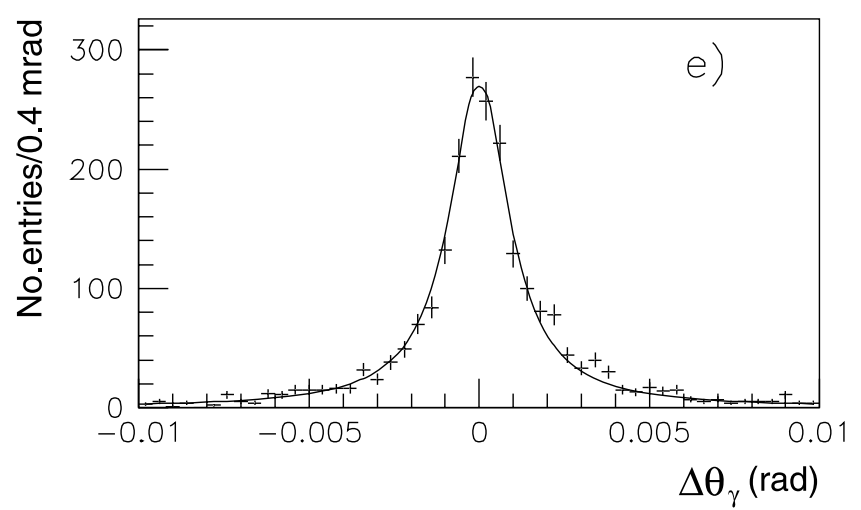

Fig. 1 (a) Distribution of the final state radiation production angle in $Z^{0} \rightarrow \mu^{+} \mu^{-}$events generated with formula (2); (b) opening angle between the generated and reconstructed directions of a muon track; (c) difference between generated and reconstructed photon polar angles $\Theta_{\gamma} ;(\mathbf{d})$ the same for the azimuthal angles $\Phi_{\gamma} ;(\mathbf{e})$ difference be-

\section{Experimental technique}

\subsection{The DELPHI detector}

The DELPHI detector is described in detail elsewhere $[23,24]$. The following is a brief description of the subdetector units relevant for this analysis.

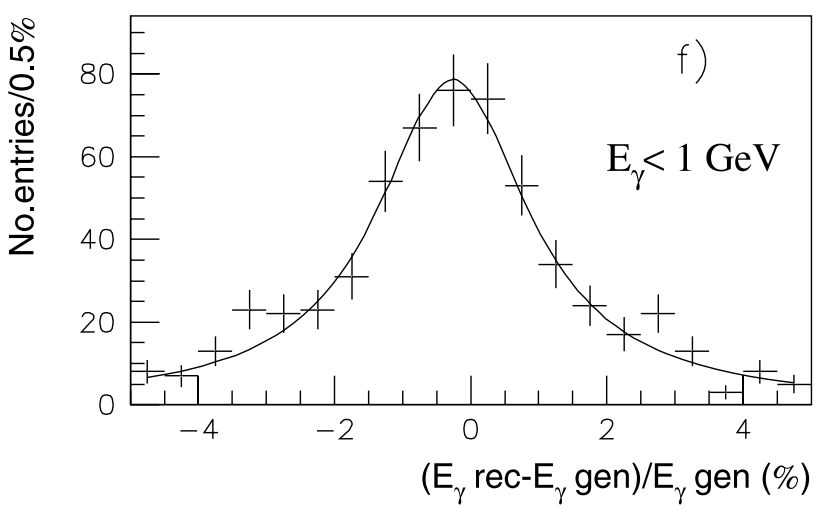

tween generated and reconstructed $\mu \gamma$ opening angles $\theta_{\gamma}$, which illustrates the overall angular resolution of this analysis; (f) difference between the generated and the reconstructed photon energies in the photon energy range of $0.2<E_{\gamma} \leq 1 \mathrm{GeV}$. The curves in Fig. 1c-1f are the fits by Breit-Wigner forms (see text)

In the DELPHI reference frame the $z$ axis is taken along the direction of the $e^{-}$beam. The angle $\Theta$ is the polar angle defined with respect to the $z$-axis, $\Phi$ is the azimuthal angle about this axis and $R$ is the distance from this axis.

The TPC, the principal device used in this analysis, was the main tracker of the DELPHI detector; it covered the an- 
gular range from $20^{\circ}$ to $160^{\circ}$ in $\Theta$ and extended from $30 \mathrm{~cm}$ to $122 \mathrm{~cm}$ in $R$. It provided up to 16 space points for pattern recognition and ionization information extracted from 192 wires. The TPC together with other tracking devices (Vertex Detector, Inner Detector, Outer Detector and Forward Chambers) ensured a very good angular accuracy of the muon track reconstruction, which is a part of the overall angular resolution for the photon production angle. The distribution of the opening angles between the generated and reconstructed muon directions is shown in Fig. 1b; it can be characterized by the distribution mean of $0.42 \mathrm{mrad}$ and its r.m.s. width of $0.37 \mathrm{mrad}$, which restricts $90 \%$ of the entries within the $0-1 \mathrm{mrad}$ interval.

The identification of muons was based on the muon chambers (MUC) surrounding the detector, the hadron calorimeter (HCAL) and the electromagnetic calorimeter (High density Projection Chamber, HPC), as described in [25-27].

The Monte Carlo (MC) data set used in this analysis was produced with the DYMU3 generator [28, 29]. Higher order radiative corrections to the reaction (1) total cross section were accounted for via the exponentiation procedure implemented in the generator. The generated dimuon events were passed through the DELPHI detector simulation program DELSIM [24].

\subsection{Detection of photons}

Photon conversions in front of the main DELPHI tracker (TPC) were reconstructed by an algorithm that examined the tracks reconstructed in the TPC. A search was made along each TPC track for the point where the tangent of the track trajectory points directly to the beam spot in the $R \Phi$ projection. Under the assumption that the opening angle of the electron-positron pair is zero, this point represented a possible photon conversion point at radius $R$. All tracks which have had a solution $R$ that was more than one standard deviation away from the main vertex, as defined by the beam spot, were considered to be conversion candidates. If two oppositely charged conversion candidates were found with compatible conversion point parameters they were linked together to form the converted photon. The following selection criteria were imposed:

- the $\Phi$ difference between the two conversion points should be at most $30 \mathrm{mrad}$;

- a possible difference between the polar angles $\Theta$ of the two tracks should be at most $15 \mathrm{mrad}$;

- at least one of the tracks should have no associated hits in front of the reconstructed mean conversion radius.

For the pairs fulfilling these criteria a $\chi^{2}$ was calculated from $\Delta \Theta, \Delta \Phi$ and the difference of the reconstructed conversion radii $\Delta R$ in order to find the best combinations in cases where there were ambiguous associations. A constrained fit was then applied to the electron-positron pair candidate which forced a common conversion point with zero opening angle and collinearity between the momentum sum and the line from the beam spot to the conversion point.

The photon detection efficiency, i.e. the conversion probability combined with the reconstruction efficiency, was determined with the hadronic MC data since the converted photon sample in dimuon events was insufficient statistically for such a determination. The efficiencies were tabulated against three variables: $E_{\gamma}, \Theta_{\gamma}$ (the photon polar angle to the beam), and $\theta_{\gamma t k}$ (the photon opening angle to the closest track). The efficiency varied with the energy from zero at the $0.2 \mathrm{GeV}$ detection threshold up to $4-6 \%$ at $E_{\gamma} \geq 1 \mathrm{GeV}$, depending on the two other variables (for details see [1]).

In order to reduce a possible difference in the reconstruction of the converted photons in the MC and the real data (originating from the bias in the detector material distributions in the two data sets and from a possible distinction in their pattern recognition results) the recalibration procedure described in [1] was implemented, with the recalibration coefficients obtained with hadronic events.

The angular precision of the photon direction reconstruction was studied using the dimuon MC events and was found to be of a Breit-Wigner shape, as expected for the superposition of many Gaussian distributions of varying width [30]. The full widths ( $\Gamma$ 's) of the $\Delta \Theta_{\gamma}$ and $\Delta \Phi_{\gamma}$ distributions were $2.3 \pm 0.1 \mathrm{mrad}$ and $1.9 \pm 0.1 \mathrm{mrad}$, respectively, for the combined $0.2-10 \mathrm{GeV}$ interval (Figs. 1c, 1d). The full width of the distribution of the difference $\Delta \theta_{\gamma}$ between the generated and reconstructed muon-photon opening angles (which is the difference in the production angle $\theta_{\gamma}$ defined in Sect. 2 and therefore represents the overall angular resolution of the current analysis) was found to be $2.1 \pm 0.1 \mathrm{mrad}$ (Fig. 1e), thus providing a possibility for the observation of the muon bremsstrahlung dead cone. Moreover, one can improve essentially this raw resolution, though at the price of a loss of 50\% of the converted photon statistics, by requiring the photon energy to exceed $1 \mathrm{GeV}$ and the conversion radius to be greater than $25 \mathrm{~cm}$. With these tighter cuts $1.4 \mathrm{mrad}$ resolution (the full width) was achieved and used in a particular case which required a high angular resolution and is described below (Sect. 7.3).

The accuracy of the converted photon energy measurement was studied also with the dimuon MC events. In both energy bands it was at the level of $\pm 1.5 \%$ (the Breit-Wigner full width about 3\%); this is illustrated by Fig. If where the distribution of the relative difference between the generated and reconstructed photon energy is plotted for the LE photons. The resolution was checked with events of the (hadronic) real data by comparing the $\pi^{0}$ peak width of the $\gamma \gamma$ mass distribution from these data to the analogous one from the MC. 


\section{Data selection}

\subsection{Selection of dimuon events}

The data selection was done under standard cuts aimed at the separation of dimuon events (cf. [17, 25-27]) which are described below. The consecutive application of these cuts reduced the MC sample of dimuon events by factors indicated in parentheses:

- the number of charged particles $N_{c h}$ had to be within the interval of $2 \leq N_{c h} \leq 5$, and the two highest momentum particles had to have $p>15 \mathrm{GeV} / c(0.894)$;

- the polar angles of the two highest momentum particles had to be within the interval of $20^{\circ} \leq \Theta \leq 160^{\circ}(0.962)$;

- the impact parameters of the two highest momentum particles had to be less than 0.2 and $4.5 \mathrm{~cm}$ in the $R \Phi$ and $z$ projections, respectively (0.993);

- no additional charged particles with momenta greater than $10 \mathrm{GeV} / c$ were allowed, unless the fastest particle had a momentum greater than $40 \mathrm{GeV} / c$ (0.999);

- the acollinearity of the two highest momentum particles had to be less than $10^{\circ}(0.989)$;

- the two highest momentum particles had to be identified as muons using either the muon chambers (MUC), the hadron calorimeter (HCAL), or the electromagnetic calorimeter (HPC), by requiring associated hits in the muon chambers, or by energy deposition in the calorimeters consistent with a minimum ionizing particle $(0.825)$.

The total reduction factor for the MC events was 0.696.

A total of 122812 events of real data (RD) was selected under these cuts and compared to 373918 selected MC events corresponding, after the normalization of the equivalent MC luminosity to the integrated RD luminosity, to 121000 expected events.

\subsection{Selection of photons}

The standard selection of converted photons was done under the following cuts:

- only converted photons with both $e^{+}, e^{-}$arms reconstructed were considered;

- $20^{\circ} \leq \Theta_{\gamma} \leq 160^{\circ}$;

- $5 \mathrm{~cm} \leq R_{\text {conv }} \leq 50 \mathrm{~cm}$, where $R_{\text {conv }}$ means conversion radius;

- $200 \mathrm{MeV}<E_{\gamma} \leq 10 \mathrm{GeV}$.

384 and 1097 converted photons were found using these cuts in the real data in the LE and HE energy bands, respectively. Of these, 127 and 265 photons are in the selected $p_{T}$ regions: $p_{T}<40 \mathrm{MeV} / c$ for the LE band and $p_{T}<80 \mathrm{MeV} / c$ for the $\mathrm{HE}$ band.
For a particular analysis done to scrutinize the dead cone effect (described in Sect. 7.3), the photon energy was required to be between 1 and $10 \mathrm{GeV}$, and the conversion radius to be between 25 and $50 \mathrm{~cm}$.

\section{Backgrounds}

The following background sources within the $\mu^{+} \mu^{-}$event sample were considered:

- External muon bremsstrahlung: the bremsstrahlung radiation from muons when they pass through the material of the experimental setup.

- Secondary photons: when a high energy photon (of any origin) generates an $e^{+} e^{-}$pair in the detector material in front of the TPC the pair particles may radiate (external) bremsstrahlung photons, which can enter our kinematic region.

- "Degraded" photons:

higher energy converted primary photons with degraded energy measurement due to the secondary emission of (external) bremsstrahlung by at least one of their electrons.

DELSIM was invoked to reproduce these processes in the MC stream.

Collection of background photons (all dubbed as External Brems) in the MC stream was done if any one of the following conditions was satisfied:

- a given photon was absent at the event generator level, i.e. in the DYMU3 event record;

- a given photon, found in the DYMU3 event record, migrated from outside a selected $p_{T}$ region into that region due to the energy degradation.

$26.0 \pm 2.9$ and $61.5 \pm 4.5$ background photons (normalized to the RD statistics) were found in the selected $p_{T}$ regions: $p_{T}<40 \mathrm{MeV} / c$ for the LE band and $p_{T}<80 \mathrm{MeV} / c$ for the HE band, respectively.

The background from $Z^{0} \rightarrow \tau^{+} \tau^{-}$events was estimated using the MC data produced with the KORALZ 4.0 generator [31] and passed through the full detector simulation and the analysis procedure. The $\tau^{+} \tau^{-}$contamination of the dimuon event sample was found to be $1536 \pm 20$ events (1.3\% of the dimuon sample), which contain zero photons in the LE band and $1.3 \pm 0.6$ photons in the HE band, of which 0.3 photons would be in the $p_{T}<80 \mathrm{MeV} / c$ region. In what follows, this background was neglected.

The cosmic ray background was estimated from the real data, studying events which originated close to the interaction point, but outside the limits allowed for selected events. In both energy bands its contribution to the photon rates was below $0.1 \%$. 
The background from $Z^{0} \rightarrow e^{+} e^{-}$events tested with the BABAMC generator [32] with the full detector simulation was found to be vanishingly small. The same is valid for the 4-fermion backgrounds $Z^{0} \rightarrow e^{+} e^{-} \mu^{+} \mu^{-}$and $Z^{0} \rightarrow \mu^{+} \mu^{-} q \bar{q}$ tested with events produced with generators [33-36].

\section{Systematic errors}

\subsection{Systematic uncertainties in the determination of the signal}

Since the converted photon sample in the dimuon event statistics collected by the DELPHI experiment during the LEP1 period was insufficient for the determination of the photon detection efficiencies and the recalibration coefficients, they were taken as being defined with hadronic events. Therefore it is worth to start the consideration of the systematic errors and their estimations with the uncertainties induced by these components of the analysis as they were determined in [1].

The uncertainty due to a difference in the photon propagation and conversion in the detector material in the RD and its simulation in the MC, and analogous difference in the pattern recognition, left after the recalibration procedure was applied (termed in [1] hardware systematics), was studied in [1] and evaluated to be $0.9 \%$ of the photon rate in the LE band and $2 \%$ in the HE band.

The systematic error for the photon detection efficiency ${ }^{3}$, after the recalibration procedure mentioned above being applied, is a purely instrumental effect originating from the choice of the binning of the variables used for the efficiency parametrization, resolution effects, etc. In [1] it was found to range from $6 \%$ to $9 \%$ of the photon rate. These estimations were tested in the current study with the MC dimuon events by comparing the $p_{T}$ spectra of the DYMU3 inner bremsstrahlung photons transported through the DELPHI detector by DELSIM with a subsequent simulation of their conversions, with the spectra of the same photons taken at the generator level and convoluted with the photon detection efficiency. In both energy bands the difference was below $5 \%$ which was the level of the test statistical accuracy. This means that the aforementioned error due to the detection efficiency is likely to be overestimated in [1], or it is really smaller in the muonic data, in particular, due to a bet-

\footnotetext{
${ }^{3}$ Note that when dealing with the data uncorrected for the detection efficiency the efficiency error is relevant to the bremsstrahlung predictions only (since bremsstrahlung spectra have to be convoluted with the efficiencies in this case). On the contrary, when dealing with the corrected data the efficiency uncertainty has to be applied to the measured photon rates only.
}

ter angular resolution and due to a narrower angular ranges in both energy bands. In what follows, the value of $5 \%$ is used as an estimate for the uncertainty of the efficiency calculation.

The systematic errors originating from the influence of the $p_{T}$ resolution on the selection cuts were estimated from runs with the reconstructed photon energy and production angle randomly shifted according to the appropriate resolution function (taking into account the different angular resolutions in the LE and HE bands). The changes were found to be less than $0.3 \%$ of the photon rate in the LE band and less than $0.4 \%$ of the photon rate in the HE band. In what follows, the corresponding errors were neglected.

The uncertainty of the background (BG) estimation is composed of the uncertainties coming from the DYMU3 generator, efficiency and hardware systematics, BG selection, and the procedure of the BG photon conversion simulation. The systematic error from the photon conversion simulation is considered to be equal to the systematic error of the photon detection in the MC stream, before the recalibration is applied, i.e. it can be approximated by the recalibration corrections, which were within $3-4 \%$. The systematic errors due to efficiency and hardware in the background estimation have strong positive correlations with the analogous components of the systematic error in the calculation of the real data photon rates (indeed they are of the same relative amplitude, but the background errors have to be reduced by factors of 3.9 and 3.3 in the LE and HE bands, respectively, when entering the final systematic error, since the background rates within the corresponding $p_{T}$ intervals constitute $25.7 \%$ and $30.2 \%$ of the RD-BG photon rates in the corresponding energy bands). Ignoring, for the sake of clarity, these correlations we will consider all the background systematics components as independent and uncorrelated with the analogous components in the RD rates. Then, calculating the background systematic errors similarly to those for the RD and taking into account the suppression factors mentioned above, the systematic background uncertainties appear to be $1.4 \%$ and $2.2 \%$ relative to the signal rate in the respective energy band in the case of the uncorrected data, and $1.9 \%$ and $2.7 \%$ in the case of the data corrected for efficiency.

The above systematic errors are summarized in Table 1.

\subsection{Estimation of the accuracy of the bremsstrahlung predictions}

The estimation of the accuracy of the bremsstrahlung predictions resulting from formula (2) was done by comparing FSR rates obtained with this formula and those delivered by the DYMU3 generator, in the corresponding $p_{T}$ ranges, as the difference between the predictions. In the LE band this uncertainty was about $4 \%$, in the HE band about $10 \%$. They are quoted in Table 1. 
Table 1 Systematic uncertainties (in \% of the photon rates in the $p_{T}$ ranges below $40 \mathrm{MeV} / c$ and $80 \mathrm{MeV} / c$ for the LE and HE photons, respectively) for the signal and the predicted muon inner bremsstrahlung. The total systematic error of each of the two energy band photon rates and signal-to-bremsstrahlung ratios is the quadratic sum of the corresponding individual errors, as quoted in Tables 2, 3 below

\begin{tabular}{|c|c|c|c|c|}
\hline \multirow[t]{2}{*}{ Component } & \multicolumn{2}{|c|}{$\begin{array}{l}\text { Data uncorrected for } \\
\text { the detection efficiency }\end{array}$} & \multicolumn{2}{|c|}{$\begin{array}{l}\text { Data corrected for } \\
\text { the detection efficiency }\end{array}$} \\
\hline & LE band & HE band & LE band & HE band \\
\hline & \multicolumn{4}{|c|}{ Signal } \\
\hline Recalibration & $0.9 \%$ & $2.0 \%$ & $0.9 \%$ & $2.0 \%$ \\
\hline Efficiency & - & - & $5.0 \%$ & $5.0 \%$ \\
\hline \multirow[t]{2}{*}{ Background } & $1.4 \%$ & $2.2 \%$ & $1.9 \%$ & $2.7 \%$ \\
\hline & \multicolumn{4}{|c|}{ Predicted Bremsstrahlung } \\
\hline Efficiency & $5.0 \%$ & $5.0 \%$ & - & - \\
\hline Formula (2) & $4.0 \%$ & $10.0 \%$ & $4.0 \%$ & $10.0 \%$ \\
\hline
\end{tabular}

These estimates agree well with the differences in the predictions for the muon inner bremsstrahlung rates obtained with formula (2), and those calculated with formulae which account for higher order radiative corrections, the calculations being performed with the non-exponentiated photon spectrum [37] and with the exponentiated one [38, 39]. In particular, the latter give $5.9 \%$ and $9.1 \%$ differences with formula (2) in the LE and HE bands, respectively. Note that when doing these calculations, parameter $\beta$ which governs the bremsstrahlung photon spectrum [22] was obtained by integration of formula (1.2) in [38] applying our $p_{T}$ cuts, i.e. within rather narrow angular ranges varying as a function of the photon energy according to the $p_{T}$ cuts imposed in the corresponding energy band. The $\beta$ values were found to be 0.0146 in the LE band and 0.0088 in the HE band, i.e. considerably smaller than $\beta=2 \alpha / \pi\left(\ln s / m_{\mu}^{2}-1\right)=0.0582$, obtained by integration over all angles. The smallness of $\beta$ reduces the difference between the bremsstrahlung predictions for the exponentiated and non-exponentiated photon spectra.

\section{Results}

Photon distributions for $\theta_{\gamma}, p_{T}$ and $p_{T}^{2}$ are presented both for the data and the background (left panels of Figs. 2-5), and for their difference (right panels of the figures). The latter distributions are accompanied by the calculated bremsstrahlung spectra according to (2) shown by triangles.

To quantify the excess of the data over the background the difference between them, which represents the measured muon inner bremsstrahlung, was integrated in the $p_{T}$ interval from 0 to $40 \mathrm{MeV} / c$ for the photons of the $\mathrm{LE}$ band, and from 0 to $80 \mathrm{MeV} / c$ for the photons of the $\mathrm{HE}$ band (these intervals correspond to the filled areas in panels $\mathrm{d}, \mathrm{f}$ of Figs. 2-5), and the values obtained were defined as signals. However these $p_{T}$ cuts were not applied when filling the angular distributions displayed in Figs. 2-6 in order to keep these distributions unbiased.

\subsection{Energy band $0.2<E_{\gamma} \leq 1 \mathrm{GeV}, p_{T}<40 \mathrm{MeV} / c$}

Photon distributions, uncorrected and corrected for the photon detection efficiency, are displayed in Figs. 2 and 3, respectively. The results for the signal rate are given in Table 2 together with the predictions for the muon bremsstrahlung and their ratios.

As can be seen from Table 2, the predicted and the measured muon bremsstrahlung rates agree well, within the measurement errors. The small differences in Signal/IB ratios between corrected and uncorrected data in Table 2 (and Table 3 below) arise from the non-uniformity of the efficiency reweighting factors.

\subsection{Energy band $1<E_{\gamma} \leq 10 \mathrm{GeV}, p_{T}<80 \mathrm{MeV} / c$}

Photon distributions, uncorrected and corrected for the photon detection efficiency, are displayed in Figs. 4 and 5, respectively. The results for the signal rate are given in Table 3 together with the predictions for the muon bremsstrahlung and their ratios.

As can be seen from Table 3, the predicted and the measured muon bremsstrahlung rates agree well, within the measurement errors. The smaller values of the corrected experimental and predicted bremsstrahlung rates in the HE energy band as compared to those in the LE band (while the energy range factor following from formula (2), $\ln \left(E_{\gamma}^{\max } / E_{\gamma}^{\text {min }}\right)$, works in favour of the HE band with an enhancement factor of 1.43) are explained by a higher attenuation of the rates induced by the $p_{T}$ cut in the case of the bremsstrahlung photons from the HE band.

\subsection{Observation of the dead cone of the muon bremsstrahlung}

The distributions of the photon production angles with a fine binning (of $1 \mathrm{mrad}$ bin width) are shown in Figs. 6a, b for the combined sample of the converted photons from both energy bands. The distribution obtained after back- 


\section{DELPHI}
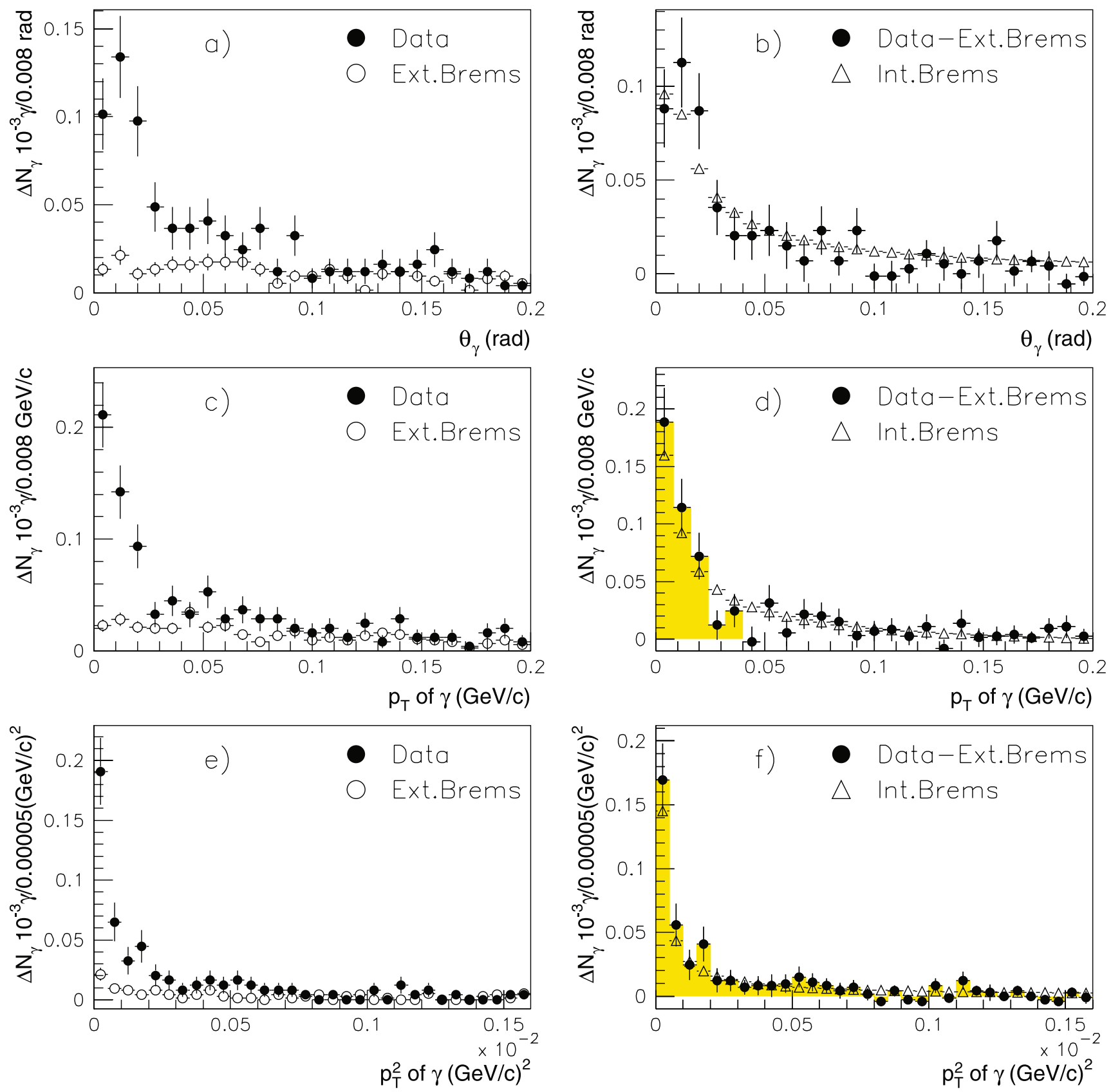

Fig. 2 Photon distributions in the photon energy band $0.2<E_{\gamma} \leq$ $1 \mathrm{GeV}$ uncorrected for the photon detection efficiency. The photon rates are given as the number of photons per 1000 muons per bin width of the distribution. Left panels: the data and background distributions for (a) $\theta_{\gamma}$, the photon production angle; (c) photon $p_{T} ;(\mathbf{e})$ photon $p_{T}^{2}$.

ground subtraction (Fig. 6b) is accompanied by the calculated bremsstrahlung points. The displayed measured distributions are raw spectra, without any unfolding of the detector angular resolution; the bremsstrahlung spectra calculated with formula (2) were smeared instead by the resolution. We prefer to present the uncorrected measured distributions in

Right panels, (b), (d), (f): the difference between the data and the background for the same variables, respectively. "Ext.Brems" corresponds to the background, "Int.Brems" corresponds to the muon inner bremsstrahlung predictions. The filled areas in panels (d) and (f) correspond to the signal integral (see text). The errors shown are statistical

order to demonstrate the independence of the obtained results on the correction procedure.

As can be seen from the plots, the experimental points follow well the predicted bremsstrahlung distribution, showing a turnover at the expected bremsstrahlung peak position of $4 \mathrm{mrad}$. This is therefore an observation of the muon inner 
DELPHI
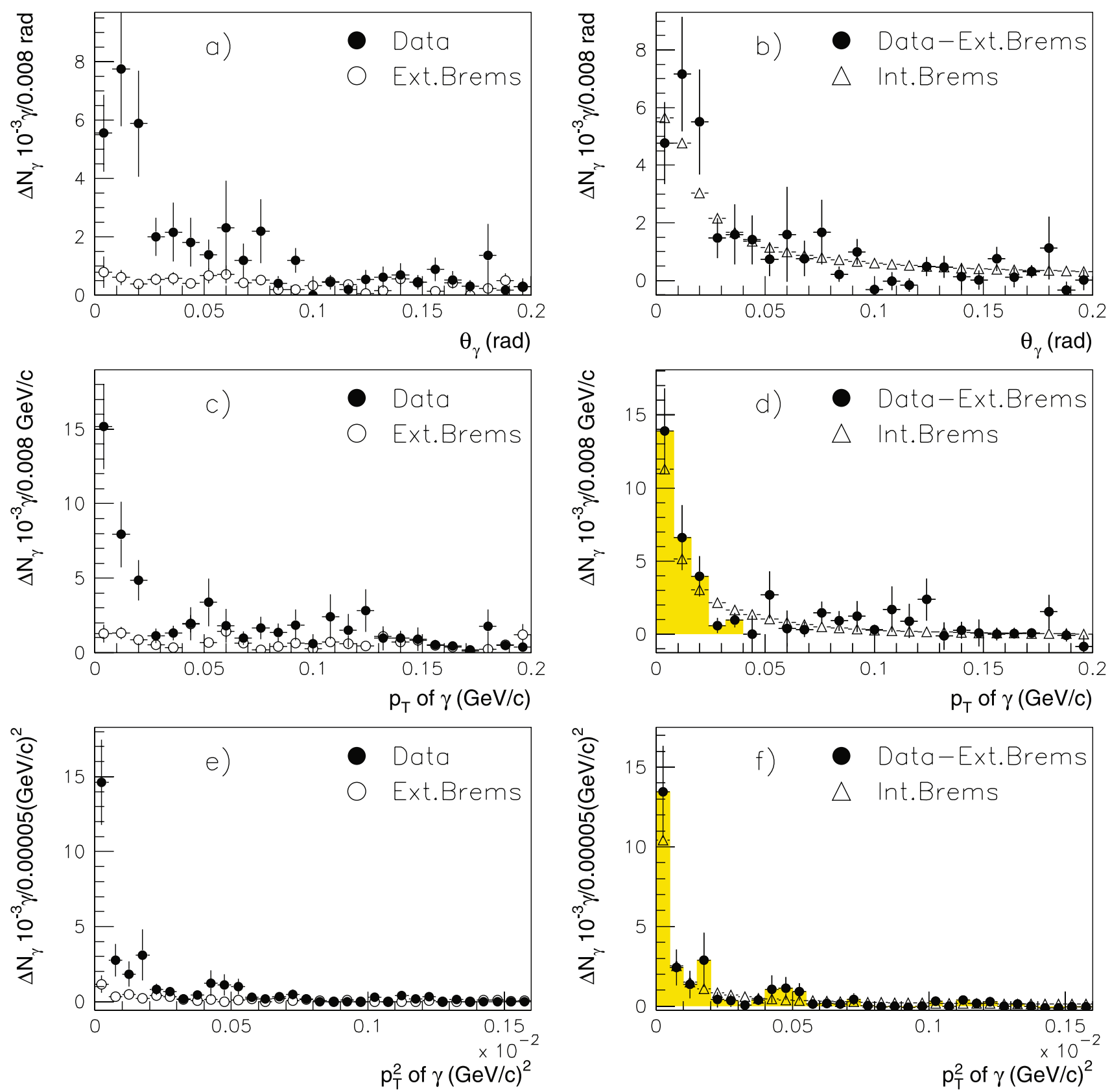

Fig. 3 The same as in Fig. 2, corrected for the efficiency of photon detection

Table 2 The signal (RD-Background), the predicted muon inner bremsstrahlung (both in units of $10^{-3} \gamma / \mu$ ) and their ratios in the $p_{T}<40 \mathrm{MeV} / c$ range for the photons from the LE band. The first errors are statistical, the second ones are systematic

\begin{tabular}{lcc}
\hline Value & Data uncorrected for the detection efficiency & Data corrected for the detection efficiency \\
\hline Signal & $0.412 \pm 0.048 \pm 0.007$ & $25.9 \pm 4.0 \pm 1.4$ \\
Inner Bremsstrahlung & $0.388 \pm 0.001 \pm 0.025$ & $23.30 \pm 0.01 \pm 0.93$ \\
Signal/IB & $1.06 \pm 0.12 \pm 0.07$ & $1.11 \pm 0.17 \pm 0.07$ \\
\hline
\end{tabular}


DELPHI
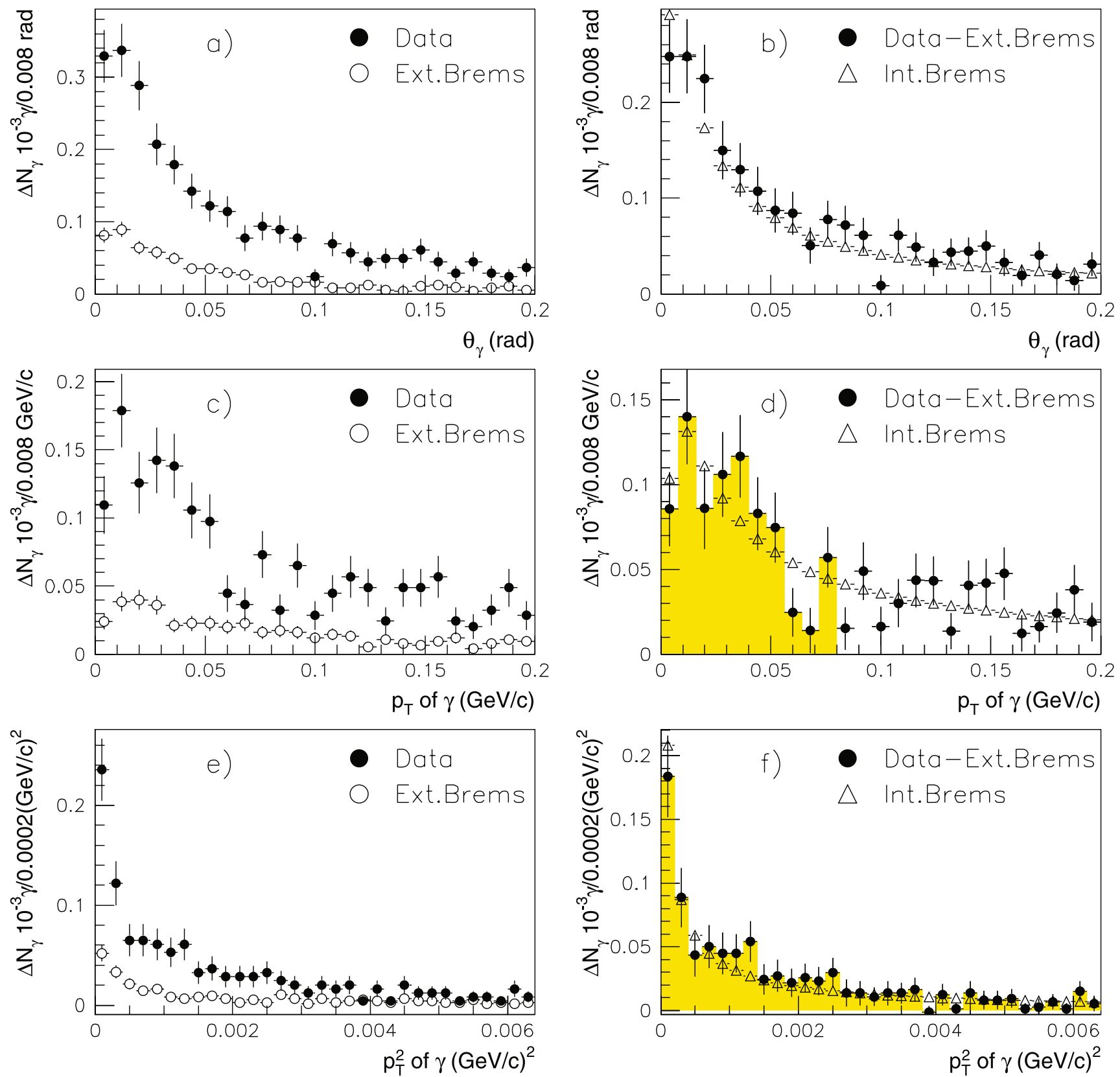

Fig. 4 Photon distributions in the photon energy band $1<E_{\gamma} \leq$ $10 \mathrm{GeV}$ uncorrected for the photon detection efficiency. The photon rates are given in number of photons per 1000 muons per bin width of the distribution. Left panels: the data and background distributions for (a) $\theta_{\gamma}$, the photon production angle; (c) photon $p_{T} ;(\mathbf{e})$ photon $p_{T}^{2}$.

Right panels, (b), (d), (f): the difference between the data and the background for the same variables, respectively. The filled areas in panels (d) and (f) correspond to the signal integral (see text). The errors shown are statistical

bremsstrahlung dead cone, for the first time in high energy physics experiments. The observation enriches the agreement between the experimental findings of the muon inner bremsstrahlung characteristics reported in this work and the QED predictions for the process.

However a deeper insight into the bremsstrahlung pattern can be obtained when considering, instead of the distri-

bution $d N_{\gamma} / d \theta_{\gamma}$, the distribution $d N_{\gamma} / d \Omega$, where $d \Omega$ is a solid angle element. Such a distribution is free of kinematic suppression at the polar angles $\theta_{\gamma}$ approaching zero, and the remaining suppression of the photon production rate at very small angles is a purely dynamic effect, similar to that mentioned in Sect. 2 for the hadrons inside a jet, namely a destructive interference between the radiation sources, but this 
DELPHI
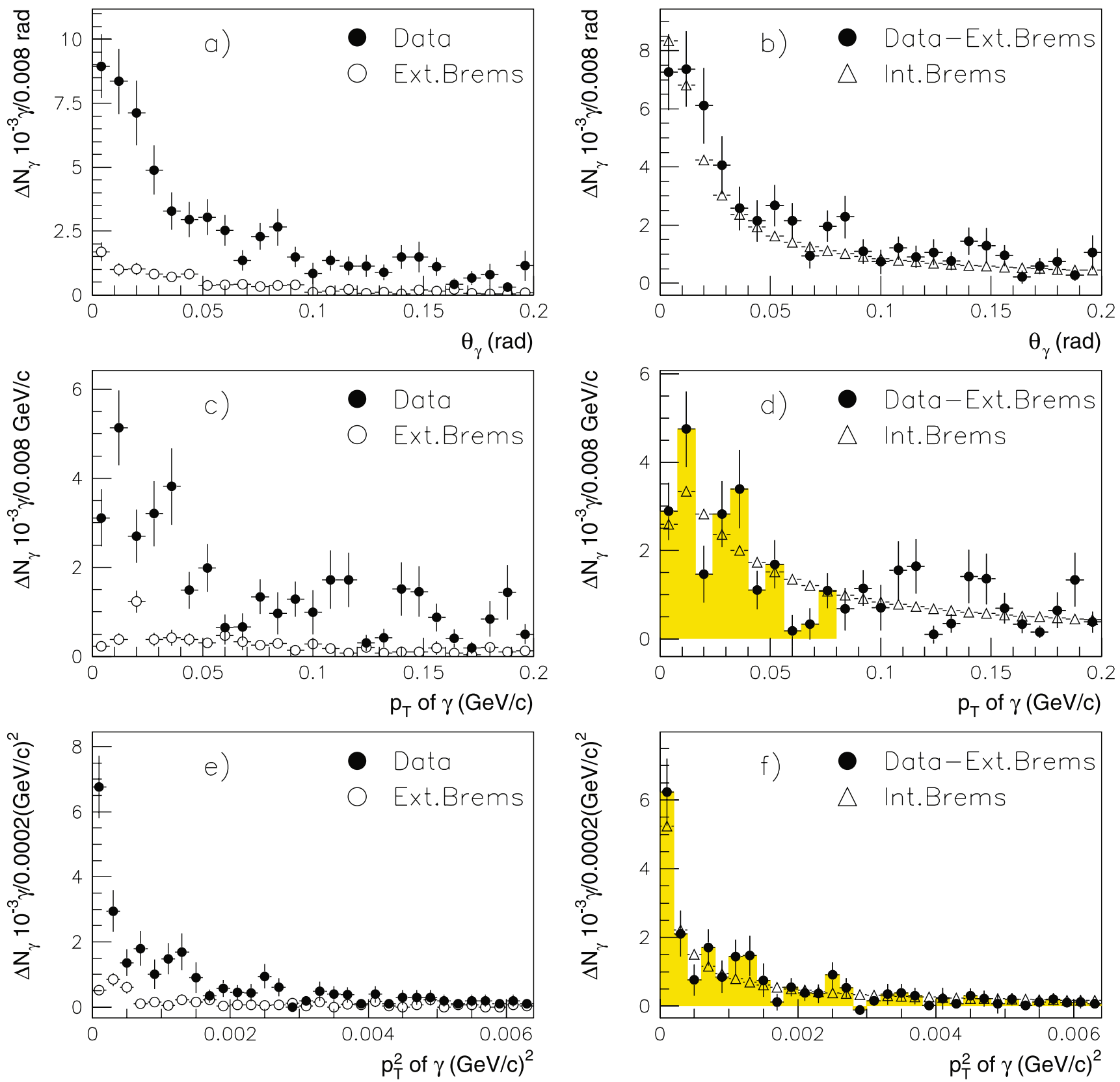

Fig. 5 The same as in Fig. 4, corrected for the efficiency of photon detection

Table 3 The signal (RD-Background), the predicted muon inner bremsstrahlung (both in units of $10^{-3} \gamma / \mu$ ) and their ratios in the $p_{T}<$ $80 \mathrm{MeV} / c$ range for the photons from the HE band. The first errors are statistical, the second ones are systematic

\begin{tabular}{lcc}
\hline Value & Data uncorrected for the detection efficiency & Data corrected for the detection efficiency \\
\hline Signal & $0.829 \pm 0.069 \pm 0.025$ & $21.1 \pm 2.2 \pm 1.3$ \\
Inner Bremsstrahlung & $0.794 \pm 0.001 \pm 0.089$ & $20.00 \pm 0.01 \pm 2.00$ \\
Signal/IB & $1.04 \pm 0.09 \pm 0.12$ & $1.06 \pm 0.11 \pm 0.12$ \\
\hline
\end{tabular}




\section{DELPHI}
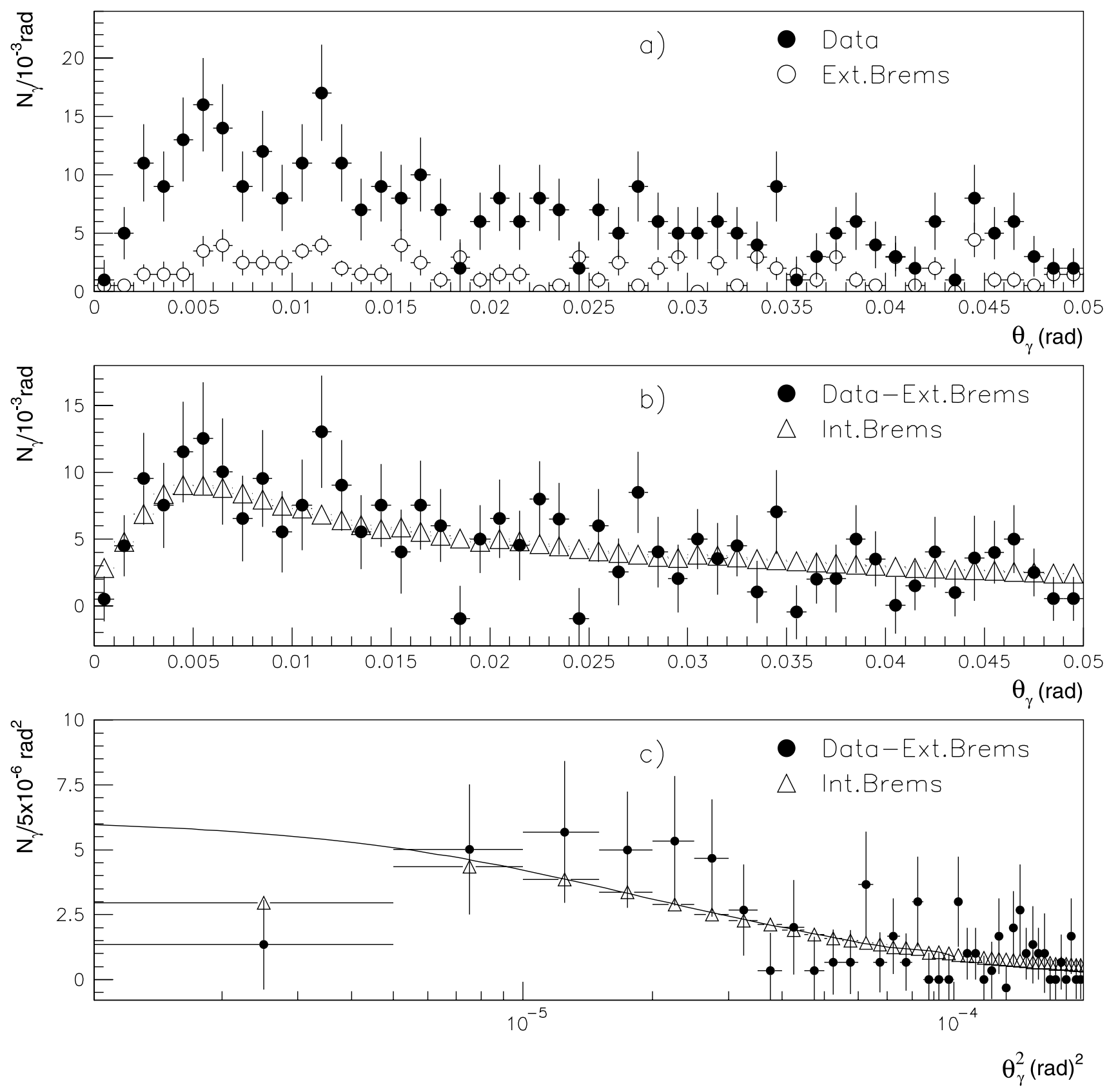

Fig. 6 Dead cone of the muon inner bremsstrahlung: $(\mathbf{a}, \mathbf{b})$ as seen in the photon production angle distributions: (a) the data and the background distributions; (b) the difference between the data and the background; and (c): the distribution of the photon production angle

time less straightforward, just between the muon "before" and "after" the photon emission. ${ }^{4}$ The solid angle element

\footnotetext{
${ }^{4}$ In classical language, the radiation intensity into the solid angle $d \Omega$ vanishes when three vectors: the muon velocity, its acceleration, and the radiation unit vector happen, in particular, to be parallel, see for example [40, 41].
}

squared, obtained under tighter cuts that improve the angular resolution; the curve shows the fit of the bremsstrahlung distribution within $10^{-5} \leq \theta_{\gamma}^{2}<10^{-4} \mathrm{rad}^{2}$ by a 5 th order polynomial extrapolated to the 1 st bin of the distribution (see text). The errors shown are statistical

$d \Omega$ is proportional to $d \cos \theta_{\gamma}$, which at small angles is, in turn, proportional to $d \theta_{\gamma}^{2}$. The position of the $d N_{\gamma} / d \theta_{\gamma}^{2}$ distribution turnover is predicted to be at $\theta_{\gamma}^{2}=1 / \Gamma^{2}(\Gamma=430$, see Sect. 2), i.e. at $\theta_{\gamma}^{2}=5.4 \times 10^{-6} \mathrm{rad}^{2}$.

To observe this turnover, an improved angular resolution was required, achieved with the additional cuts (see Sect. 4.2) to be at the level of $1.4 \mathrm{mrad}$, as noticed in 
Sect. 3.2. The distribution $d N_{\gamma} / d \theta_{\gamma}^{2}$ obtained with this resolution is shown in Fig. 6c, together with the bremsstrahlung predictions for this variable. Though the statistics are poor, the dip at $\theta_{\gamma}^{2}<5 \times 10^{-6} \mathrm{rad}^{2}$ is visible in this distribution, revealing the dynamical dead cone of the muon inner bremsstrahlung.

In order to estimate the statistical significance of this observation the following procedure was undertaken. The initial part (about 20 bins) of the bremsstrahlung $\theta_{\gamma}^{2}$ distribution shown in Fig. 6c, with first two bins omitted, was fitted by a smooth curve (by a polynomial of 4 th or 5 th order). Then the fitting curve was extrapolated to zero, as shown in the figure giving the value of $(5.64 \pm 0.27) \gamma / 5 \times 10^{-6} \mathrm{rad}^{2}$ at the centre of the first bin of the distribution (the error reflects the variation in the fitting form and in the number of bins used in the fit). This value was assumed to represent the expected bremsstrahlung rate in the first bin of the distribution in a hypothetical situation when the bremsstrahlung dynamical dead cone is absent. The number of the real data photons in the first bin was 2 with the estimated background to be $0.66 \pm 0.46$, thus giving the signal value in this bin $(1.34 \pm 1.49) \gamma / 5 \times 10^{-6} \mathrm{rad}^{2}$. Assuming Poisson distribution for the signal photons these numbers correspond to the probability of the absence of the bremsstrahlung dead cone of less than $4 \%$.

\section{Comparison with the hadronic soft photon analysis}

The main difference between the results of this analysis and the hadronic ones [1] is the absence of any essential excess of the soft photon production over the predicted inner bremsstrahlung rate reported in this study, contrary to the case for [1] where the observed soft photon rate was found to exceed the bremsstrahlung predictions by a factor of about 4 . The $95 \%$ CL upper limits on the excess factors which can be extracted from the results of this work are 1.29 in the LE band, and 1.28 in the HE band.

Another distinction between the two analyses is an essential difference in the background levels and in the possible systematic effects. However, the code transporting photons through the DELPHI detector and simulating their conversions in the detector material (DELSIM), the photon reconstruction algorithm and the determination of its efficiency, together with the recalibration procedure, were common to the two analyses. Thus the results of this work can be considered also as a cross-check of these procedures in the hadronic events study. On the other hand, the amount of dimuon events collected during the LEP1 period is considerably smaller than the number of collected hadronic events, due to a smaller $Z^{0}$ dimuon branching ratio (by a factor of 20). As a result, in the current analysis the statistical errors are either essentially higher than the systematic ones (in the LE band), or comparable to them (in the HE band), while in [1] the total uncertainties of the measured photon rates are dominated by systematic errors; nevertheless it should be emphasized that the results of both analyses show clear signals of direct photons (even though the strength of the signal in [1] is not explained theoretically).

\section{Summary}

The results of the analysis of final state radiation in $\mu^{+} \mu^{-}$ decays of $Z^{0}$ events at LEP1 are reported in this work. The radiation was studied in the region of small transverse momenta with respect to the parent muon, $p_{T}<40 \mathrm{MeV} / c$ in the photon energy range $0.2<E_{\gamma} \leq 1 \mathrm{GeV}$ (LE band), and $p_{T}<80 \mathrm{MeV} / c$ in the photon energy range $1<E_{\gamma} \leq$ $10 \mathrm{GeV}$ (HE band). The obtained photon rates uncorrected (corrected) for the photon detection efficiency were found to be, in units of $10^{-3} \gamma / \mu$, with the first error to be statistical and the second one systematic: a) in the LE band: $0.412 \pm 0.048 \pm 0.007(25.9 \pm 4.0 \pm 1.4)$, while QED predictions for the muon inner bremsstrahlung were calculated to be $0.388 \pm 0.001 \pm 0.025(23.30 \pm 0.01 \pm 0.93)$; b $)$ in the HE band: $0.829 \pm 0.069 \pm 0.025(21.1 \pm 2.2 \pm 1.3)$, while the muon inner bremsstrahlung was calculated to be $0.794 \pm 0.001 \pm 0.089(20.00 \pm 0.01 \pm 2.00)$. The obtained ratios of the observed signal to the predicted level of the muon inner bremsstrahlung are then $1.06 \pm 0.12 \pm 0.07$ in the LE band and $1.04 \pm 0.09 \pm 0.12$ in the HE band (uncorrected rates are used for these ratios, as they possess a better statistical accuracy). Thus, the analysis shows a good agreement between the observed photon production rates and the QED predictions for the muon inner bremsstrahlung, both in differential (see Figs. 2-5) and integral (see Tables 2, 3) forms. This is in contrast with the anomalous soft photon production in hadronic decays of $Z^{0}$ reported earlier in [1].

The bremsstrahlung dead cone is observed for the first time in the direct photon production in $Z^{0}$ decays in particular, and in the muon inner bremsstrahlung in the high energy physics experiments in general, also being in good agreement with the predicted bremsstrahlung behaviour.

Acknowledgements We thank Profs. K. Boreskov, J.E. Campagne, F. Dzheparov and A. Kaidalov for useful discussions.

We are greatly indebted to our technical collaborators, to the members of the CERN-SL Division for the excellent performance of the LEP collider, and to the funding agencies for their support in building and operating the DELPHI detector.

We acknowledge in particular the support of

Austrian Federal Ministry of Education, Science and Culture, GZ 616.364/2-III/2a/98,

FNRS-FWO, Flanders Institute to encourage scientific and technological research in the industry (IWT) and Belgian Federal Office for Scientific, Technical and Cultural affairs (OSTC), Belgium, FINEP, CNPq, CAPES, FUJB and FAPERJ, Brazil, Ministry of Education of the Czech Republic, project LC527, 
Academy of Sciences of the Czech Republic, project AV0Z10100502, Commission of the European Communities (DG XII),

Direction des Sciences de la Matière, CEA, France,

Bundesministerium für Bildung, Wissenschaft, Forschung und Technologie, Germany,

General Secretariat for Research and Technology, Greece,

National Science Foundation (NWO) and Foundation for Research on

Matter (FOM), The Netherlands,

Norwegian Research Council,

State Committee for Scientific Research, Poland, SPUB-M/CERN/ PO3/DZ296/2000, SPUB-M/CERN/PO3/DZ297/2000, 2P03B 10419 and 2P03B 69 23(2002-2004),

FCT-Fundação para a Ciência e Tecnologia, Portugal,

Vedecka grantova agentura MS SR, Slovakia, Nr. 95/5195/134,

Ministry of Science and Technology of the Republic of Slovenia,

CICYT, Spain, AEN99-0950 and AEN99-0761,

The Swedish Research Council,

Particle Physics and Astronomy Research Council, UK,

Department of Energy, USA, DE-FG02-01ER41155,

EEC RTN contract HPRN-CT-00292-2002.

\section{References}

1. J. Abdallah et al., DELPHI Collaboration, Eur. Phys. J. C 47, 273 (2006)

2. P.V. Chliapnikov et al., Phys. Lett. B 141, 276 (1984)

3. F. Botterweck et al., Z. Phys. C 51, 541 (1991)

4. S. Banerjee et al., Phys. Lett. B 305, 182 (1993)

5. A. Belogianni et al., Phys. Lett. B 408, 487 (1997)

6. A. Belogianni et al., Phys. Lett. B 548, 122 (2002)

7. A. Belogianni et al., Phys. Lett. B 548, 129 (2002)

8. L.D. Landau, I.Ya. Pomeranchuk, Dokl. Akad. Nauk SSSR 92, 535 and 735 (1953) (Papers No. 75 and 76 in the English edition of L.D. Landau collected works, Pergamon Press, New York, 1965)

9. F. Low, Phys. Rev. 110 , 974 (1958)

10. V.N. Gribov, Sov. J. Nucl. Phys. 5, 280 (1967)

11. V. Balek, N. Pišútová, J. Pišút, Acta Phys. Pol. B 21, 149 (1990)

12. P. Lichard, Phys. Rev. D 50, 6824 (1994)

13. J. Abdallah et al., DELPHI Collaboration, Study of the dependence of direct soft photon production on the jet characteristics in hadronic $Z^{0}$ decays, Eur. Phys. J. C, to be submitted

14. P. Abreu et al., DELPHI Collaboration, Eur. Phys. J. C 16, 371 (2000)

15. J. Abdallah et al., DELPHI Collaboration, Eur. Phys. J. C 45, 589 (2006)
16. J. Abdallah et al., DELPHI Collaboration, Eur. Phys. J. C 46, 295 (2006)

17. P. Abreu et al., DELPHI Collaboration, Z. Phys. C 65, 603 (1995)

18. P.D. Acton et al., OPAL Collaboration, Phys. Lett. B 273, 338 (1991)

19. P. Abreu et al., DELPHI Collaboration, Phys. Lett. B 380, 480 (1996)

20. H.C. Ballagh et al., Phys. Rev. Lett. 50, 1963 (1983)

21. V.V. Ammosov et al., Sov. J. Nucl. Phys. 47, 73 (1988)

22. J. Haissinski, How to compute in practice the energy carried away by soft photons to all orders in $\alpha$, LAL 87-11, 1987; http://ccdb4fs.kek.jp/cgi-bin/img_index?8704270

23. P. Aarnio et al., DELPHI Collaboration, Nucl. Instrum. Methods A 303, 233 (1991)

24. P. Abreu et al., DELPHI Collaboration, Nucl. Instrum. Methods A 378, 57 (1996)

25. P. Abreu et al., DELPHI Collaboration, Nucl. Phys. B 367, 511 (1991)

26. P. Abreu et al., DELPHI Collaboration, Nucl. Phys. B 417, 3 (1994)

27. P. Abreu et al., DELPHI Collaboration, Nucl. Phys. B 418, 403 (1994)

28. J.E. Campagne, R. Zitoun, Z. Phys. C 43, 469 (1989)

29. J.E. Campagne et al., in $Z$ Physics at LEP1, ed. by G. Altarelli, R. Kleiss, C. Verzegnassi, CERN Yellow Report No. 89-08, 1989, vol. 3, 3.2.5

30. W.T. Eadie et al., Statistical Methods in Experimental Physics (North-Holland, Amsterdam, 1982), p. 90

31. S. Jadach, B.F.L. Ward, Z. Was, Comput. Phys. Commun. 79, 503 (1994)

32. F.A. Berends, R. Kleiss, W. Hollik, Nucl. Phys. B 304, 712 (1988)

33. F.A. Berends, R. Pittau, R. Kleiss, Comput. Phys. Commun. 85, 437 (1995)

34. T. Sjöstrand, Comput. Phys. Commun. 39, 347 (1986)

35. T. Sjöstrand, M. Bengtsson, Comput. Phys. Commun. 43, 367 (1987)

36. T. Sjöstrand, JETSET 7.3 Program and Manual, CERN-TH/648892, 1992

37. F.A. Berends, R. Kleiss, S. Jadach, Nucl. Phys. B 202, 63 (1982)

38. D.R. Yennie, S.C. Frautschi, H. Suura, Ann. Phys. 13, 379 (1961)

39. R. Kleiss et al., in $Z$ Physics at LEP1, ed. by G. Altarelli, R. Kleiss, C. Verzegnassi, CERN Yellow Report No. 89-08, 1989, vol. $3,2.2$

40. L.D. Landau, E.M. Lifshitz, The Classical Theory of Fields, 4th revised English edn. (Elsevier, Amsterdam), Sect. 73

41. J.D. Jackson, Classical Electrodynamics, 3rd edn. (Willey, New York), Sect. 14.3 